\title{
Grading of Urothelial Carcinoma and The New "World Health Organisation Classification of Tumours of the Urinary System and Male Genital Organs 2016”
}

Eva M. Compérat ${ }^{a, *}$, Maximilian Burger ${ }^{b}$, Paolo Gontero ${ }^{c}$, A. Hugh Mostafid ${ }^{d}$, Joan Palou ${ }^{e}$, Morgan

Rouprêt ${ }^{\text {f,g }}$, Bas W.G. van Rhijn ${ }^{\text {h }}$, Shahrokh F. Shariat ${ }^{\text {, }}$, Richard J. Sylvester ${ }^{j}$, Richard Zigeuner ${ }^{k}$, Marko Babjuk'

a Department Pathology, University Pierre et marie curie, Assistance Publique-Hôpitaux de Paris, Hopital Tenon, Paris, France

${ }^{\mathrm{b}}$ Department of Urology, Caritas St. Josef Medical Centre, University of Regensburg, Regensburg, Germany

c Urology Clinic, Citta della Salute e Della Scienza di Torino, University of Studies of Turin, Turin, Italy

${ }^{\mathrm{d}}$ Department of Urology, North Hampshire Hospital, Basingstoke, Hampshire, UK

e Department of Urology, Fundació Puigvert, Universidad Autónoma de Barcelona, Spain

${ }^{f}$ AP-HP, Hopital Pitié-Salpétrière, Service d'Urologie, Paris, France

gUPMC University Paris 06, GRC5, ONCOTYPE-Uro, Institut Universitaire de Cancérologie, Paris, France

${ }^{\mathrm{h}}$ Department of Surgical Oncology (Urology), Netherlands Cancer Institute, Antoni van Leeuwenhoek Hospital, Amsterdam, The Netherlands

i Medical University of Vienna, Vienna General Hospital, Vienna, Austria

${ }^{j}$ European Association of Urology Guidelines Office, Brussels, Belgium

${ }^{k}$ Department of Urology, Medical University of Graz, Graz, Austria

'Department of Urology, Hospital Motol, Second Faculty of Medicine, Charles University, Praha,

Czech Republic

* Corresponding author. Department of Pathology, AP-HP, University Pierre and Marie Curie, Hôpital Tenon 4, Rue de la Chine, 75020 Paris, France. Tel. +33 156017306; Fax: +33 156016496.

E-mail address: evacomperat@gmail.com (E.M. Compérat).

Keywords: Urothelial carcinoma; Classification system; Reproducibility; Prognosis; WHO;

ISUP 


\begin{abstract}
Context: In the management of urothelial carcinoma, determination of the pathological grade aims at stratifying tumours into different prognostic groups to allow evaluation of treatment results, and optimise patient management. This article reviews the principles behind different grading systems for urothelial bladder carcinoma discussing their reproducibility and prognostic value.

Objective: This paper aims to show the evolution of the World Health Organisation (WHO) grading system, discussing their reproducibility and prognostic value, and evaluating which classification system best predicts disease recurrence and progression. The most optimal classification system is robust, reproducible, and transparent with comprehensive data on interobserver and intraobserver variability. The WHO published an updated tumour classification in 2016, which presents a step forward, but its performance will need validation in clinical studies.
\end{abstract}

Evidence acquisition: Medline and EMBASE were searched using the key terms WHO 1973, WHO/International Society of Urological Pathology 1998, WHO 2004, WHO 2016, histology, reproducibility, and prognostic value, in the time frame 1973 to May 2016. The references list of relevant papers was also consulted, resulting in the selection of 48 papers. Evidence synthesis: There are still inherent limitations in all available tumour classification systems. The WHO 1973 presents considerable ambiguity for classification of the G2 tumour group and grading of the G1/2 and G2/3 groups. The 2004 WHO classification introduced the concept of low-grade and high-grade tumours, as well as the papillary urothelial neoplasm of low malignant potential category which is retained in the 2016 classification. Furthermore, while molecular markers are available that have been shown to contribute to a more accurate histological grading of urothelial carcinomas, thereby improving selection of treatment for a given patient, these are not (yet) part of standard clinical practice.

Conclusions: The prognosis of patients diagnosed with urothelial carcinoma greatly depends on correct histological grading of the tumour. There is still limited data regarding intraobserver and interobserver variability differences between the WHO 1973 and 2004 classification systems. Additionally, reproducibility remains a concern: histological differences between the various types of tumour may be subtle and there is still no consensus amongst pathologists. The recent WHO 2016 classification presents a further improvement on the 2004 classification, but until further data becomes available, the European Association of Urology currently recommends the use of both WHO 1973 and WHO 2004/2016 classifications. 
Patient summary: Bladder cancer, when treated in time, has a good prognosis. However, selection of the most optimal treatment is largely dependent on the information your doctor will receive from the pathologist following evaluation of the tissue resected from the bladder. It is therefore important that the classification system that the pathologist uses to grade the tissue is transparent and clear for both urologists and pathologists. A reliable classification system will ensure that aggressive tumours are not misinterpreted, and less aggressive cancer is not overtreated. 


\section{Introduction}

This article reviews the principles behind different grading systems for urothelial bladder carcinoma and discusses their reproducibility and prognostic value. It will not address urinary cytology, as this paper's focus is on histological criteria. The European Association of Urology (EAU) Nonmuscle Invasive Bladder (NMIBC) Guidelines Panel recommends the use of both the World Health Organisation (WHO) 1973 and WHO 2004/2016 classifications [1]. This article touches on the strengths and weaknesses of the available classifications. The aim of tumour classification is to be able to identify groups of patients according to different outcomes and manage them accordingly. An ideal classification system will be robust, reproducible, and transparent. It should be relatively easy for pathologists to understand and apply, and for urologists and oncologists to utilise in the treatment decisionmaking process.

During the last $40 \mathrm{yr}$, different bladder tumour-grading systems have been introduced, with generally good acceptance. One of the most widely accepted systems has been the WHO 1973 system, which is still used in some parts of Europe [2]. However, the currently recommended system is the new WHO 2016 grading system, which is being proposed for universal use and should be adopted worldwide [3].

\subsection{The WHO 1973 classification}

The WHO 1973 grading system was proposed by Mostofi et al [2] and differentiates papillary urothelial lesions into three grades: G1, G2, and G3 (Table 1) [4]. Tumours are graded according to the degree of cellular and architectural atypia. The lowest grade (G1) displays nearly no atypia, while the highest grade (G3) displays major atypia with major architectural disorders, such as loss of polarity or pseudostratification. The WHO 1973 is a robust and time-tested grading system. Some centres still prefer it to newer grading systems because it allows a comparison of long-term outcome results between different clinical centres. The major limitation of the WHO 1973 classification is the G2 group of tumours.

\subsubsection{G1 tumours}

In G1 tumours, the papillary urothelial carcinomas consist of orderly-arranged, nearly-normal, urothelial cells. The tumours display slender papillae with minimal abnormality. Mitosis and necrosis are globally absent. Nuclear pleomorphism is absent, with regular chromatin distribution. If present, nucleoli are small. Cell borders are well defined. 


\subsubsection{G3 tumours}

In G3 tumours, architectural and nucleocytoplasmic heterogeneity is a typical finding. Mitosis can be distributed all over the urothelium, even in superficial layers, and necrosis may be present. There is increased cellularity, nuclear crowding, and a lack of normal differentiation. The nuclei display irregular membranes and shape and sometimes neoplastic giant cells. Chromatin distribution is granular or coarse and prominent nucleoli are common.

\subsubsection{G2 tumours}

In the G2 group of tumours, several different types of noninvasive urothelial carcinomas are grouped broadly together. The architectural and nucleocytoplasmic atypia in G2 cover a broad spectrum of lesions between those seen in grades G1 and G3. The variation in polarity and nuclear aspects can be very important for treatment decision making and even though subclassification of grades was never recommended in the WHO 1973 classification [5], some authors have tried to subclassify G2 tumours into G2a and G2b subgrades on the basis of nuclear polymorphism and mitotic count [6,7].

In addition, the WHO 1973 system has led some pathologists to ambiguously grade tumours that fall between grades 1 and 2 as G1/2, and tumours that fall between grades 2 and 3 as G2/3. Grade G1/2 subgroups contain relatively low-grade (LG) G2 urothelial carcinomas, which are much closer to G1, and relatively high-grade (HG) tumours, which are much closer to G3. This practice creates further ambiguity, complicates the treatment decision-making process and should therefore be avoided [7].

\subsection{The WHO/International Society of Urological Pathology 1998 classification system}

In 1997, a new multidisciplinary consensus meeting was held to revisit terminology and provide updated recommendations to the WHO on the pathology of urothelial carcinomas. The WHO/International Society of Urological Pathology (ISUP) classification of 1998 distinguishes papilloma, papillary urothelial neoplasm of low malignant potential (PUNLMP), and LG and HG carcinomas. This classification system was the first to introduce the category of PUNLMP [4]. PUNLMP was subsequently included in the WHO 2004 classification and, although initially controversial [8], is retained in the WHO 2016 classification without any polemic [3].

The distinction made between PUNLMP and LG urothelial carcinoma is based on morphology. While PUNLMP and LG urothelial carcinoma differ on a molecular level, few papers address this aspect. A paper by Barbisan et al [9] described overexpression of FGFR3 
and CK20 on a protein level in urothelial neoplasm of low malignant potential, while another paper by Lee et al [10] found lower expression levels of microRNAs-125b in LG noninvasive bladder cancer compared with PUNLMPs. These findings underline why the distinction between both entities is necessary since some pathologists consider PUNLMP to be completely benign, while others consider PUNLMPs to belong to the LG pTa group. The WHO/ISUP 1998 grading system also introduced a description and definition of hyperplasia and flat lesions of the bladder.

\subsection{The WHO 2004/2016 classification systems}

The WHO 2016 system is based on the WHO/ISUP 1998 classification and the WHO 2004 classification (Fig. 1) [8], which refined the criteria of WHO/ISUP 1998 [4]. The division of urothelial tumours into LG and HG tumours, as introduced in the WHO 2004 classification, remains part of the WHO 2016 system.

According to the WHO 2016 system, pTa and pT1 tumours are graded into LG and HG and all detrusor muscle-invasive urothelial carcinomas are considered to be HG tumours. pTa tumours do not invade the lamina propria (no lymphovascular invasion and distant metastasis). However, pT1 tumours do grow under the basement membrane into the lamina propria, and lymphovascular invasion and metastasis can be seen in these cases. In many instances pathologists will identify pT1 tumours as HG tumours, independently of their atypia.

Strong points of the 2016 classification are clear cut-offs between HG and LG tumours, along with clear, precise descriptions of each grade in order to obtain homogeneous groups of tumours. The system includes three distinct categories: PUNLMP, pTa LG, and pTa HG. It avoids the use of ambiguous grading, G1/2 or G2/3, that fail to place a tumour in a defined grade.

The term noninvasive has also been introduced in the WHO 2016 system to further differentiate noninvasive LG and HG papillary carcinoma from invasive urothelial carcinomas. Consequently, the classification of pTa HG carcinomas in the WHO 2016 system is expansive enough to encompass all HG tumours with similar biological properties. This is a clear advantage of the WHO 2016 compared with the WHO 1973 classification, where these tumours could be randomly distributed between $\mathrm{G} 2$ and 3 .

\subsubsection{WHO 2016 grades and histological groups}

1.3.1.1. Papillary urothelial neoplasms of low malignant potential 
The PUNLMP category is useful because it does not carry the name cancer. This is important in younger patients, in whom PUNLMP mostly presents, who would otherwise have to carry a diagnosis of cancer with all its psychological and financial consequences. If the criteria for PUNLMP are applied in the very strictest way the classification only pertains to a very restricted group of tumours. The papillae of this exophytic lesion are discreet, slender, and not fused, covered by mostly normal urothelial cells, with absent or minimal cytological atypia. There is a slight increase in the number of atypic cells, polarity is preserved, and nuclei can be increased when compared with normal tissue. Umbrella cells are mostly present. Mitosis, if present, is the exception, and only found in the basal layers. Necrosis is absent. The prognosis of these lesions is very good. Although there is undeniably a risk of recurrence, the risk of progression is low (Table 2).

It should be noted that in 2002 PUNLMP was a new, very controversial entity, and pathologists, as well as urologists, had to get used to it on a diagnostic level. Therefore, as pathologists became more familiar with the entity over time, the intraobserver and interobserver reproducibility improved and cohorts became more robust. As a result, it is likely that the rates found in later studies are more accurate due to an improved understanding of PUNLMP and the recruitment of more consistent cohorts.

Reports of the progression rate of PUNLMP are consistent: it was reported as $8 \%$ by Pan et al [11] in 2010, 8\% in 2002 by Samaratunga et al [12], and 10\% in 2015 by Compérat et al [13]. In Pich et al [14], the median disease-free survival was 76 mo and no patient with PUNLMP showed progression towards a higher-grade tumour. Similar findings were reported by Holmang et al [15], with no progression in his study of 95 patients.

All these data, considered together, show that PUNLMP are not completely benign lesions, may recur and, according to some published data, can show progression, even though some authors dispute the difference between PUNLMP and pTa LG tumours and consider them to belong in the same tumour group (Table 2). The management of PUNLMPs is not yet clear and further research is required to determine follow-up and length of surveillance.

Meanwhile, long-term follow-up is recommended, similar to that for pTa tumours.

\subsection{Noninvasive papillary $L G$ urothelial carcinoma}

LG pTa carcinoma is characterised by orderly-arranged papillae. Although variations in polarity and nuclear size, shape and chromatin distribution are not of primary importance, a specific cytological disorder exists. Mitosis is rare, and, if present, usually occurs in the lower half of the urothelium. The challenges presented by inclusion and tangential cutting may 
make it difficult to analyse architectural aspects, which can be mistaken for irregular urothelium, while fused glands may be overgraded. However, if different grades are detected in the same lesion, the tumour should be graded according to the highest grade observed. It should be noted that the histological differences between PUNLMP and LG pTa can sometimes be subtle.

\subsection{Noninvasive papillary $\mathrm{HG}$ urothelial carcinoma}

Some HG lesions are characterised by a completely disorderly appearance at low magnification due to both cytonuclear and architectural disorganisation. The spectrum of pleomorphism is wide and ranges from moderate to marked, but consensus is to not subdivide this group of lesions into two subgroups. However, the degree of nuclear atypia is important: nuclei have prominent nucleoli and are pleomorphic, with frequent mitosis. Intraepithelial necrosis can be present. The thickness of urothelium can vary considerably; the papillae are fused and display an anarchic growth.

HG tumours must be considered to be aggressive lesions. HG tumours can be pTa, but also pT1-4. Urothelial carcinomas pT2-4 are generally classified as HG tumours according to the WHO 2004/2016 [3,8].

\subsection{Carcinoma in situ}

Carcinoma in situ (CIS) is considered to be a HG lesion. It has very variable characteristics. McKenney et al [16] described four different morphological aspects: large-cell pleomorphic type, large-cell nonpleomorphic, small-cell, which does not refer to a neuroendocrine differentiation, but describes cells smaller than in the large-cell group, and clinging denuded form. Also described are three types of cancerisation of the urothelium, where only parts of the urothelium show major atypia. These types are: pagetoid form, with single atypical cancerous cells in the urothelium, the undermining form, in which the basal layer is replaced by highly atypical cells showing all the characteristics of HG lesions, and the overriding form, where the upper layer is replaced [16].

CIS is often observed in the adjacent mucosa of pTa HG lesions. At a molecular level, HG pTa carcinomas resemble invasive urothelial carcinomas. Comparative genomic hybridisation studies have demonstrated deletions at 2q, 5q, 10q, and 18q and gains at 5q and 20q. HG tumours show a high level of progression of $50-65 \%$ and a recurrence rate of $14.8-80.7 \%$ [17-19]. 


\section{Evidence acquisition}

\subsection{Intraobserver and interobserver variability and reproducibility}

A major challenge for every histological grading system is the degree to which the subjectivity of the pathologist affects the classification. When introducing a new grading system, the comparison is always delicate, as pathologists must move away from the old, familiar, system and try to adopt the new one.

\subsection{Comparison of reproducibility of WHO 1973 and WHO 2004 classification systems}

Pathologists who work together show better reproducibility when grading tumours [20]. Furthermore, reproducibility is much greater when pathologists are using a familiar grading system, rather than a new one. A recent meta-analysis compared both the WHO 1973 and WHO 2004 systems for performance and reproducibility. The authors concluded that the intra- and interobserver variability was slightly less in the 2004/2016 classification, but that it did not show a better prediction of recurrence and progression [21].

The main criticism concerning the reproducibility of the 1973 WHO classification is the poorly-defined morphological criteria. No distinct cut-offs between the different tumour grades were suggested, and there existed a clear lack of reproducibility. The variability of G2 tumours ranged from $13 \%$ to $69 \%$ and the lack of reproducibility has been a major argument in favour of rethinking the grading system [7,21]. The inclusion of ambiguous grading such as G1/2 and G2/3 gave no clear guidance to clinicians on the course of treatment for such patients.

\subsubsection{Recurrence and progression}

\subsubsection{1. $\quad$ pTa tumours}

Several studies have tried to compare the classifications for progression and recurrence with the ultimate aim of finding practice changing results. Cao et al [22] examined $172 \mathrm{pTa}$ carcinomas with good follow-up and demonstrated significant differences between LG and HG papillary carcinomas in recurrence-free survival $(p=0.01)$. The authors concluded that the 2004 WHO system was superior to the 1973 system for predicting clinical outcomes in patients with urothelial carcinoma, independent of pathologic stage, and underlined the usefulness in pTa tumours. Yin and Leong [23] demonstrated significant differences in recurrence between LGand HG pTa carcinomas ( $p<0.5$ ), but none with the WHO 1973 classification. The study of May et al [24] compared the prognostic implications of both WHO systems and showed a significant difference in 5-yr recurrence rate between LG and 
HG pTa carcinomas, but no significant difference in 5-yr progression-free survival (PFS). In a similar way, G1-3 WHO 1973 pTa tumours had significantly different 5-yr recurrence-free survival (RFS) rates as well as PFS.

Lokeshwar et al [25] reported a significant grade shift in pTa bladder cancers with the use of the WHO 2004 classification, which did not seem to correlate with disease progression. They pointed out the problem of both tumour categories being treated with a range of different treatments, as well as the frequent overtreatment of patients.

\subsubsection{2. $\quad \mathrm{pT} 1$}

Only a few studies comparing the different grading systems in pT1 carcinomas exist. There seems to be some evidence that the 1973 WHO classification might add some benefit to predict survival of patients [22,26,27]. However, the study of Cao et al [22] failed to show a difference for the RFS, PFS, and oveeall survival between pT1 G2 and G3 carcinomas, while the study of van Rhijn et al [27] showed that G2 and G3 played a significant role as prognosticator towards muscle invasive carcinomas. The paper of Otto et al [26] showed that the WHO 1973 was a good predictor for disease-specific survival in pT1 bladder carcinomas. Campbell et al [28] demonstrated moderate interobserver variability $(\kappa=0.6)$ in the case of two pathologists when comparing bladder tumours according to the WHO 1998 classification. In a study of 258 consecutive papillary urothelial carcinomas, Gonül et al [29] showed a correlation between tumour grades in both WHO 1973 and WHO 2004/2016 classification systems (Table 3). Overall agreement between the two pathologists in the study was higher using the WHO 2004/2016 classification than with the WHO 1973 system. Again, PUNLMP showed the lowest degree of agreement. If the diagnosis of PUNLMP was excluded, the kappa ( $\kappa)$ value increased from 0.41 to 0.84 [11].

\subsection{Intraobserver variability}

Pellucchi et al [30] reported the evaluation of 270 consecutive patients with a first episode of LG pTa tumours. The same pathologist determined the grade for each tumour as LG pTa and G1 or G2 according to the WHO 1973 and WHO 2004 classification systems, respectively. The 5-yr PFS was 93\% for the LG pTa group, 97.6\% for the G1 group, and 93.3\% for the G2 group, while the 5-yr RFS was $49.4 \%$ for the LG group, $62 \%$ for the G1 group, and $40 \%$ for the G2 group. They concluded that the WHO 1973 system was a better predictor of recurrence than the WHO 2004 system, but that both systems predicted the risk of progression with similar accuracy [13]. 
The WHO 2004 classification showed better reproducibility than the WHO 1973 system, with the lowest agreement achieved with PUNLMP versus LG pTa. Further training could have a positive impact on the grading of these lesions, especially in the PUNLMP category. HG lesions were the easiest to classify with less intra- and interobserver variability.

\subsection{Heterogeneous lesions}

Not all urothelial lesions are homogeneous and grade heterogeneity is common. A mixture of two grades in the same tumour have been reported in 3-43\% of tumours $[31,32]$. The WHO/ISUP 1998 consensus meeting suggested reporting the highest grade of a lesion as, at that time, no published evidence existed on this topic [4]. The WHO 2004 system also recommended basing the grade of a heterogeneous tumour on the highest grade observed [8]. Furthermore, the arbitrary criterion to ignore a finding of less than 5\% of HG tumour has an unknown impact. Very little research has been carried out to confirm these suggestions for grading [32].

Based on the 1998 WHO/ISUP classification system, Cheng et al [33] suggested a modified grading system and that the volume of a grade should be $>5 \%$ to be considered for grading [16].

A recent paper from Gofrit et al [34] showed that 5\% of resections had mixed grade tumours which he defined as LG tumours with $10 \%$ or less of a HG component. As long as the HG component is not higher than $10 \%$, these tumours have an evolution like LG carcinomas. According to the hypothesis that normal looking urothelium adjacent to CIS and tumours accumulates neoplastic molecular changes [35], the study by Downes et al [35] evaluated those mixed tumours at the morphologic, proteonomic, and molecular levels. The authors demonstrated that LG areas in mixed tumours already exhibited molecular changes associated with disease progression. These findings suggest that molecular changes in HG tumours occur at an early stage of tumour growth, even before the corresponding morphological features become visible, which underlines the current practice of grading papillary urothelial neoplasms based on the highest grade [35].

The distinction between LG and HG tumours is of major importance. In the case of borderline histology, other parameters such as urinary cytology, multifocality, size of the lesion, prior history, and recurrence can play a role in grading. If CIS is present, the tumour should be considered to be a HG lesion. Currently, no authoritative recommendations exist on how to report mixed lesions; neither the WHO 2016, nor the International Collaboration on Cancer Reporting (www.iccr-cancer.org), provide a clear statement on the subject. Using cut-offs 
(5\% according to Cheng [33] 10\% according to Gofrit [34]) is arbitrary and not validated; therefore, the pathologist should record a percentage and describe all morphological aspects.

\section{Evidence synthesis}

\section{1. $\quad$ Molecular grading}

Urothelial carcinomas are often heterogeneous cell populations, with different morphological aspects and clinical outcomes. Although grading is a major prognostic factor, its usefulness needs to be improved by stronger intra- and interobserver reproducibility in determining tumour grade.

Molecular markers have a role in grading and their use may help to produce a more accurate diagnosis. FGFR-3 and P53 (TP53) mutations have been recognised as key pathways in the genetic development of urothelial neoplasias. In the early 2000s, the first studies combining FGFR-3 and grading were published. They confirmed an association between FGFR-3 mutation and LG tumours ( $p<0.0001$ ), with FGFR-3 detected in 84\% of G1 tumours [29]. FGFR-3 is one of the most frequently mutated oncogene in urothelial carcinomas. It seems to be associated with LG tumours, noninvasive stage, and low recurrence. In contrast, P53 mutations are linked to a high-tumour grade, advanced stage, and frequent recurrences. Molecular markers can help to refine the accuracy of a histological diagnosis.

\subsection{Taxonomy and gene signatures in NMIBC}

Since 2012 molecular grading has been refined, Sjödahl et al [36] introduced a five-group system, with the Urobasal group A, which is predominantly made of pTa LG tumours, clearly demonstrating better outcomes. Furthermore, Sjödahl et al [36] also demonstrated that NMIBC still have a urothelial differentiation, which they lose as they become more aggressive. In 2015, Patschan et al [37] analysed pT1 carcinomas and demonstrated a molecular subtype classification with urobasal (32\%), genomically unstable (58\%), and squamous cell carcinoma-like (10\%) [37]. They also showed that rapidly progressing T1 tumours were of subtype genomically unstable or squamous cell carcinoma-like and had high lymphocyte (CD3) levels. Another paper, by Yun et al [38], may show that molecular risk classifiers predict the risk of progression and response to Bacillus Calmette-Guérin and could help to identify the optimal management of HG T1 disease for each patient. Those pT1 carcinomas which progress seem to have a molecular signature close to pT2 carcinomas. Due to the still limited data on NMIBC, no internationally accepted recommendations exist for 
molecular prognostic or grading markers. Current management will rely on pathologist assessment, based on morphological aspects.

\subsection{Markers}

\subsubsection{Tumour recurrence}

A study by Compérat et al [39] compared the different grades of pTa tumours and the expression of MIB-1 and aurora kinase A, which are markers for early mitosis. The study found that aurora kinase A and MIB-1 combined with the WHO 2004 grading system formed a powerful predictor of tumour recurrence.

Another study found an FGFR-3 mutation in all G1 and G2 tumours and also showed that patients with the mutation developed fewer recurrences $(p=0.004)$. van Rhijn et al [40] suggested a molecular grading system in tumours with an FGFR-3 mutation in combination with $M I B-1$ expression. The study showed that FGFR-3 mutation was present in $88 \%$ of G1 tumours, but in only $16 \%$ of G3 tumours. The authors concluded that molecular grading was a relatively simple and reproducible method.

\subsubsection{Tumour progression}

A study by van Oers et al [41] used tissue microassays to investigate the expression of Ki-67, p53, and CK20, as markers for disease progression, and SNaPshot analysis to detect FGFR-3 mutation. Abnormal CK20 staining was strongly associated with higher tumour grades. In a group of pTaG1 tumours, 59\% presented with abnormal CK20 staining, whereas $82 \%$ carried the FGFR-3 mutation. In a group of bladder tumours with a normal CK20 pattern, the FGFR3 gene was mutated in $89 \%$, whereas FGFR-3 mutations were only found in $37 \%$ of cases with abnormal CK20 staining. All markers were strong predictors for survival in univariate studies. In multivariate analysis, they were not independent from classical pathological parameters. None of the molecular markers was significantly associated with tumour recurrence [28].

The same research group also performed a prospective study linking the WHO 1973 and 2004 grading systems to $\mathrm{Ki}-67$ and $\mathrm{CK} 20$ expression and to FGFR-3 mutation [42]. The study showed that both grading systems remained statistically significant independent predictors of progression $(p=0.005)$. However, the combination of WHO 2004 grade and FGFR-3 mutation status allowed improved risk stratification for patients with HG nonmuscle-invasive tumours [43]. 
Van Rhijn et al [44] proposed an alternative grading to validate the European Organisation for Research and Treatment of Cancer risk score, taking into account both $M I B-1$ expression and FGFR-3 mutation status. FGFR-3 mutations were significantly related to favourable disease. However, MIB-1 overexpression was seen in pT1 HG tumours with high European Organisation for Research and Treatment of Cancer risk scores [44].

It is clear that molecular markers can add benefit to a molecular grading system and research is ongoing. Until such data are available, no authoritative recommendations can be made. At the moment these, and other, markers are under investigation and only few specialised centres use them in daily practice [45-47]. None is recommended in the EAU guidelines [1].

The latest research in molecular classification suggests that markers might eventually become more important for determining prognosis and treatment than tumour grading. Sjödahl et al [48] has used molecular classification to identify five subtypes of tumours, while Choi et al [49] has identified three subgroups, which will impact treatment decisions. It should be noted that more data are available for muscle-invasive bladder cancer.

\subsection{New concepts in pT1 HG tumours}

The WHO 2016 classification system divides pT1 tumours into LG and HG tumours, but it is increasingly recognised that most pT1 tumours are probably HG [3]. The decision to stratify pT1 carcinoma as LG was taken because while they often recur they show progression in only $5 \%$ of cases [4].

However, tumours that invade the lamina propria have an aggressive potential and can develop metastasis. In addition, it is difficult to avoid either up-staging or down-staging these lesions, as shown in a recent study by Compérat et al [20], which highlighted the difficulty of a group of experienced genitourinary pathologists in detecting an invasive tumour. Kappa $(\kappa)$ score for interobserver reproducibility ranged from 0.42 to 0.6 (mean: 0.49 ), demonstrating the difficulty of staging correctly.

The WHO 2016 includes substaging for pT1 tumours, which is a better prognostic factor than grade. The International Collaboration on Cancer Reporting committee recommends the provision of some measurement of volume, or depth of invasion (depth in $\mathrm{mm}$ ), and/or total maximum dimension of invasive tumour $(\mathrm{mm})$ and/or invasion superficial to, or involving, the muscularis mucosa, and/or deep to muscularis mucosae (the last item is equivalent to $\mathrm{pT} 1 \mathrm{a} / \mathrm{b})$. 
At a molecular level, it has been proven that these lesions are mostly aneuploid, although diploid tumours may exist. FGFR-3 mutations are common. Recurrence is relatively frequent, and about $10 \%$ of patients will show $50 \%$ progression towards a higher grade of tumour or stage of disease. Disease-specific mortality between $0.5 \%$ and $4 \%$ has been reported. In the WHO 1973 system, invasive urothelial carcinomas can theoretically be considered as G1-3 [4]. It is agreed that no invasive carcinoma can be G1. Since these neoplasms are very heterogeneous, it is easily possible for sampling to miss the presence of higher-grade carcinoma during biopsy.

Finally, it should be noted that muscle-invasive bladder cancers should always be considered HG.

\subsubsection{Histological subtypes with deceptively-benign aspects}

These types of urothelial carcinomas are sometimes difficult to stage, because they display a bland invasive front. It is important to recognise them, but also to be aware of the problems in confirming an eventual invasion, especially in case of superficial, detrusor lacking biopsies. Some of these tumour variants have very bland aspects, although they are aggressive tumours, which is probably one of the reasons why, historically, some invasive urothelial carcinomas were considered LG.

\subsubsection{Nested carcinoma}

Nested carcinoma was first described in 1979 by Stern [50], who did not realise it was malignant. It took $10 \mathrm{yr}$ for nested carcinoma to be recognised as malignant.

Many pathologists considered the nested variant to be a LG tumour because of its low mitotic activity and mild pleomorphic aspects. In case of only a few tumour nests, which are not invading the detrusor muscle, this may be the case. However, this lesion has been reported to have an aggressive behaviour, and should be considered HG according to the WHO 2004 classification $[8,20,51]$.

\subsubsection{Urothelial carcinoma with small tubules and microcystic form}

A similar problem exists for urothelial carcinoma with small tubules and the microcystic form. Although only a few cases have been reported, and it is therefore difficult to make recommendations, these carcinomas should be considered aggressive as soon as they have invaded the lamina propria [52]. 


\subsubsection{Inverted variant of urothelial carcinoma}

Urothelial carcinoma with inverted papilloma-like endophytic growth also belong to the group of benign-looking carcinomas. Borders are regular and pathologists may have the impression of tangential cutting. Furthermore, solid pushing borders can mask the invasiveness of the tumour, even while it is destroying the bladder wall, and even muscle-invasive tumours can be difficult to recognise. Nevertheless, these carcinomas are aggressive as soon as they invade the lamina propria and must be considered as HG [53].

One of these pushing inverted entities, known as large-nested urothelial carcinoma, has been described by Cox and Epstein [54]. Atypia in this entity are nearly completely absent.

However, this tumour can invade the detrusor muscle and perivesical fat and is considered to be a HG tumour (Figs. 2 and 3) [55].

\section{Conclusions}

Bladder cancer grading classifications are not perfect. The EAU NMIBC Guidelines recommend that both the WHO 1973 and WHO 2004/20016 classifications should be used [1], until further validation of the 2004/2016 grading classification. The WHO 1973 and the 2004/2016 systems each have their advantages and disadvantages. A recent systematic review by the NMIBC Guidelines Panel confirmed these findings [21].

A major advantage of the 2004/2016 classification system is that it avoids ambiguous grades and the groups are clearly classified by histological descriptions. This may be why one can expect a higher intraobserver reproducibility in due time.

The clinical behaviour of NMIBC is closely related to the tumour differentiation/grade. The addition of molecular markers will be a step forward on the path to more personalised medicine. It is important that urologists understand the strengths and weaknesses of the classification used to grade their specimens. Pathologists and urologists are partners in providing the best treatment for patients, and are required to work closely together by sharing experience and research.

Author contributions: Eva M. Compérat had full access to all the data in the study and takes responsibility for the integrity of the data and the accuracy of the data analysis.

Study concept and design: Compérat. Acquisition of data: Compérat, Babjuk. Analysis and interpretation of data: Compérat, Babjuk, Burger, Palou, Mostafid, Rouprêt, van Rhijn, Shariat, Sylvester, Zigeuner.

Drafting of the manuscript: Compérat. 
Critical revision of the manuscript for important intellectual content: Compérat, Babjuk, Burger, Palou, Mostafid, Rouprêt, van Rhijn, Shariat, Sylvester, Zigeuner.

Statistical analysis: None.

Obtaining funding: None.

Administrative, technical, or material support: None.

Supervision: Compérat.

Other: None.

Financial disclosures: Eva M. Compérat certifies that all conflicts of interest, including specific financial interests and relationships and affiliations relevant to the subject matter or materials discussed in the manuscript (eg, employment/affiliation, grants or funding, consultancies, honoraria, stock ownership or options, expert testimony, royalties, or patents filed, received, or pending), are the following: Burger, company consultant for Astellas, BMS, Ipsen Pharma, Janssen, Janssen, Pfizer, Springer, Thieme, Cephid, and Merk Sharp Dome, company speaker honorarium for Astellas, BMS, Ipsen Pharma, Janseen, trial participation in Photocure SA, Ipsen Pharma, Pfizer, Springer, Thieme, and Bayer; Palou, company consultant for Olympus, Allergan, and IBSA, company speaker honorarium for SANOFIPASTEUR, General Electric, and Combat Medical, trial participation for Presurgy; Rouprêt, company consultant for Lilly, GSK, Ipsen, Astellas, Takeda, and Sanofi Pasteur, trial participation for GSK, Pfizer, and Roche, company speaker honourarium for Roche and Zambon; van Rhijn, company consultant for Astellas 2015; Shariat, company consultant for Astellas, Olympus, Wolff, Ipsen, Cephid, and Janseen, compant speaker honorarium for Lilly, Astellas, Ipsen, Olympus, Wolff, and Janssen, trial participation for Alere Inc. trials on NMP22, Roche, MSD, and BMS, participation in a company sponsered speaker's bureau for BMS, MSD, Ipsen, Olympus, and Roche, receipt of honouraria or consultation fees from Astellas, Olympus, Wolff, Ipsen, Janssen, and Roche, receipt of grants/research supports from Astellas and Sanofi, patents: 2001 Shariat S. and Slawin K. Methods to determine prognosis after therapy for prostate cancer. U.S. Patent Application Serial Number: Docket\#60/266,976. Filed May 31, 2001, 2001 Shariat S., Lerner S. and Slawin K. Methods to determine prognosis after therapy for bladder cancer. U.S. Patent Application Serial Number: Docket\#675.003US1. Filed June 1, 2001, 2002 Shariat S., Slawin K., Kattan M., and Scardino P. Pre- and posttreatment nomograms for predicting recurrence in patients with clinically localized prostate cancer that includes the blood markers interlukin-6 soluble receptor and transforming growth, 2003 Slawin K., Kattan M., Shariat S., Stephenson A., and Scardino P. Nomogram for predicting outcome of salvage radiotherapy for suspected local recurrence of prostate cancer after radical prostatectomy. U.S. Patent Applcation Serial Number: Docket\#. Filed 2005; Zigeuner, company speaker honorarium for Pfizer, Bayer Healthcare, Roche, Novartis, Glaxo Smithkline, Amgen, and Ipsen, company consultant for Pfizer, received fellowship and travel grants from Bayer Healthcare, Pfizer, Amgen, Novartis, Glaxo Smithkline, Astellas, and Takeda, receipt of grants/reasearch support from Bayer Healthcare; Mostafid, company consultant for Kyowa Hakko UK, company speaker honourarium for Prostakan, spouse/partner in Combat Medical, and trial participation for MDS; Babjuk, company consultant for Astellas and Ipsen, company speaker honourarium for Ferring and GSK, trial participation for Sotio.

Funding/Support and role of the sponsor: None.

\section{References}


[1] Babjuk M, Bohle A, Burger M, et al. EAU Guidelines on Nonmuscle-invasive Urothelial Carcinoma of the Bladder: update 2016. Eur Urol 2017;71:447-61.

[2] Mostofi FK SL, Torloni H. Histologic typing of urinary bladder tumors: international histological classification of tumors. Geneva, Switzerland: World Health Organization; 1973.

[3] Moch H, Humphrey PA, Ulbright TM, Reuter VE. WHO Classification of Tumours of the Urinary System and Male Genital Organs. Geneva, Switserland: WHO Press; 2016.

[4] Epstein JI, Amin MB, Reuter VR, Mostofi FK. The World Health

Organization/International Society of Urological Pathology consensus classification of urothelial (transitional cell) neoplasms of the urinary bladder. Bladder Consensus Conference Committee. Am J Surg Pathol 1998;22:1435-48.

[5] Cheng L, Cheville JC, Neumann RM, Bostwick DG. Flat intraepithelial lesions of the urinary bladder. Cancer 2000;88:625-31.

[6] Malmstrom PU, Busch C, Norlen BJ. Recurrence, progression and survival in bladder cancer. A retrospective analysis of 232 patients with greater than or equal to 5-year follow-up. Scand J Urol Nephrol 1987;21:185-95.

[7] Pauwels RP, Schapers RF, Smeets AW, Debruyne FM, Geraedts JP. Grading in superficial bladder cancer. (1). Morphological criteria. Br J Urol 1988;61:129-34.

[8] Eble JN, Sauter G, Epstein JI, Sesterhenn IA. World Health Organization Classification of Tumours. Pathology and Genetics of Tumours of the Urinary System and Male Genital Organs. Lyon, France: IARC Press; 2004.

[9] Barbisan F, Mazzucchelli R, Santinelli A, et al. Immunohistochemical evaluation of global DNA methylation and histone acetylation in papillary urothelial neoplasm of low malignant potential. Int J Immunopathol Pharmacol 2008;21:615-23.

[10] Lee H, Jun SY, Lee YS, Lee HJ, Lee WS, Park CS. Expression of miRNAs and ZEB1 and ZEB2 correlates with histopathological grade in papillary urothelial tumors of the urinary bladder. Virchows Arch 2014;464:213-20.

[11] Pan CC, Chang YH, Chen KK, Yu HJ, Sun CH, Ho DM. Prognostic significance of the $2004 \mathrm{WHO} / \mathrm{ISUP}$ classification for prediction of recurrence, progression, and cancer-specific mortality of non-muscle-invasive urothelial tumors of the urinary bladder: a clinicopathologic study of 1515 cases. Am J Clin Pathol 2010;133:788-95.

[12] Samaratunga H, Makarov DV, Epstein JI. Comparison of WHO/ISUP and WHO classification of noninvasive papillary urothelial neoplasms for risk of progression. Urology 2002;60:315-9.

[13] Compérat E, Larre S, Roupret M, et al. Clinicopathological characteristics of urothelial bladder cancer in patients less than 40 years old. Virch Arch 2015;466:589-94.

[14] Pich A, Chiusa L, Formiconi A, Galliano D, Bortolin P, Navone R. Biologic differences between noninvasive papillary urothelial neoplasms of low malignant potential and low-grade (grade 1) papillary carcinomas of the bladder. Am J Surg Pathol 2001;25:1528-33.

[15] Holmäng S, Johansson SL. Stage Ta-T1 bladder cancer: the relationship between findings at first followup cystoscopy and subsequent recurrence and progression. J Urol 2002;167:1634-7.

[16] McKenney JK, Gomez JA, Desai S, Lee MW, Amin MB. Morphologic expressions of urothelial carcinoma in situ: a detailed evaluation of its histologic patterns with emphasis on carcinoma in situ with microinvasion. Am J Surg Pathol 2001;25:356-62.

[17] Hurle R, Pasini L, Lazzeri M, et al. Active surveillance for low-risk non-muscle-invasive bladder cancer: mid-term results from the Bladder cancer Italian Active Surveillance (BIAS) project. BJU Int 2016;118:935-9.

[18] Breyer J, Shalekenov S, Aziz A, et al. Increased proliferation as independent predictor of disease recurrence in initial stage pta urothelial bladder cancer. Bladder Cancer 2017;3:17380 . 
[19] Heney NM, Ahmed S, Flanagan MJ, et al. Superficial bladder cancer: progression and recurrence. J Urol 1983;130:1083-6.

[20] Compérat E, Egevad L, Lopez-Beltran A, et al. An interobserver reproducibility study on invasiveness of bladder cancer using virtual microscopy and heatmaps. Histopathology 2013;63:756-66.

[21] Soukup V, Capoun O, Cohen D, et al. Prognostic performance and reproducibility of the 1973 and 2004/2016 World Health Organization Grading Classification Systems in Nonmuscle-invasive Bladder Cancer: A European Association of Urology Non-muscle Invasive Bladder Cancer Guidelines Panel Systematic Review. Eur Urol 2017;72:801-13. [22] Cao D, Vollmer RT, Luly J, et al. Comparison of 2004 and 1973 World Health Organization grading systems and their relationship to pathologic staging for predicting longterm prognosis in patients with urothelial carcinoma. Urology 2010;76:593-9.

[23] Yin H, Leong AS. Histologic grading of noninvasive papillary urothelial tumors: validation of the $1998 \mathrm{WHO} / \mathrm{ISUP}$ system by immunophenotyping and follow-up. Am J Clin Pathol 2004;121:679-87.

[24] May M, Brookman-Amissah S, Roigas J, et al. Prognostic accuracy of individual uropathologists in noninvasive urinary bladder carcinoma: a multicentre study comparing the 1973 and 2004 World Health Organisation classifications. Eur Urol 2010;57:850-8. [25] Lokeshwar SD, Ruiz-Cordero R, Hupe MC, Jorda M, Soloway MS. Impact of 2004 ISUP/WHO classification on bladder cancer grading. World J Urol 2015;33:1929-36. [26] Otto W, Denzinger S, Fritsche HM, et al. The WHO classification of 1973 is more suitable than the WHO classification of 2004 for predicting survival in pT1 urothelial bladder cancer. BJU Int 2011;107:404-8.

[27] van Rhijn BW, van Leenders GJ, Ooms BC, et al. The pathologist's mean grade is constant and individualizes the prognostic value of bladder cancer grading. Eur Urol 2010;57:1052-7.

[28] Campbell PA, Conrad RJ, Campbell CM, Nicol DL, MacTaggart P. Papillary urothelial neoplasm of low malignant potential: reliability of diagnosis and outcome. BJU Int 2004;93:1228-31.

[29] Gonül, II, Poyraz A, Unsal C, Acar C, Alkibay T. Comparison of 1998 WHO/ISUP and 1973 WHO classifications for interobserver variability in grading of papillary urothelial neoplasms of the bladder. Pathological evaluation of 258 cases. Urol Int 2007;78:338-44. [30] Pellucchi F, Freschi M, Ibrahim B, et al. Clinical reliability of the 2004 WHO histological classification system compared with the 1973 WHO system for Ta primary bladder tumors. J Urol 2011;186:2194-200.

[31] Mai KT, Flood TA, Williams P, Kos Z, Belanger EC. Mixed low- and high-grade papillary urothelial carcinoma: histopathogenetic and clinical significance. Virchow Arch 2013;463:575-81.

[32] Reis LO, Taheri D, Chaux A, Guner G, et al. Significance of a minor high-grade component in a low-grade noninvasive papillary urothelial carcinoma of bladder. Human Pathol 2016;47:20-5.

[33] Cheng L, Neumann RM, Nehra A, Spotts BE, Weaver AL, Bostwick DG. Cancer heterogeneity and its biologic implications in the grading of urothelial carcinoma. Cancer 2000;88:1663-70.

[34] Gofrit ON, Pizov G, Shapiro A, et al. Mixed high and low grade bladder tumors--are they clinically high or low grade? J Urol 2014;191:1693-6.

[35] Downes MR, Weening B, van Rhijn BW, Have CL, Treurniet KM, van der Kwast TH. Analysis of papillary urothelial carcinomas of the bladder with grade heterogeneity: supportive evidence for an early role of CDKN2A deletions in the FGFR3 pathway. Histopathology 2017;70:281-9. 
[36] Sjödahl G, Eriksson P, Liedberg F, Hoglund M. Molecular classification of urothelial carcinoma: global mRNA classification versus tumour-cell phenotype classification. J Pathol 2017;242:113-25.

[37] Patschan O, Sjodahl G, Chebil G, et al. A molecular pathologic framework for risk stratification of stage T1 urothelial carcinoma. Eur Urol 2015;68:824-32; discussion 35-6. [38] Yun SJ, Kim SK, Kim WJ. How do we manage high-grade T1 bladder cancer? Conservative or aggressive therapy? Investig Clin Urol 2016;57(Suppl 1):S44-51. [39] Compérat E, Camparo P, Haus R, et al. Aurora-A/STK-15 is a predictive factor for recurrent behaviour in non-invasive bladder carcinoma: a study of 128 cases of non-invasive neoplasms. Virchows Arch 2007;450:419-24.

[40] van Rhijn BW, Zuiverloon TC, Vis AN, et al. Molecular grade (FGFR3/MIB- 1$)$ and EORTC risk scores are predictive in primary non-muscle-invasive bladder cancer. Eur Urol 2010;58:433-41.

[41] van Oers JM, Wild PJ, Burger M, et al. FGFR3 mutations and a normal CK20 staining pattern define low-grade noninvasive urothelial bladder tumours. Eur Urol 2007;52:760-8.

[42] Burger M, van der Aa MN, van Oers JM, et al. Prediction of progression of non-muscleinvasive bladder cancer by WHO 1973 and 2004 grading and by FGFR3 mutation status: a prospective study. Eur Urol 2008;54:835-43.

[43] Murphy WM, Takezawa K, Maruniak NA. Interobserver discrepancy using the 1998 World Health Organization/International Society of Urologic Pathology classification of urothelial neoplasms: practical choices for patient care. J Urol 2002;168:968-72.

[44] Bol MG, Baak JP, Buhr-Wildhagen S, et al. Reproducibility and prognostic variability of grade and lamina propria invasion in stages Ta, T1 urothelial carcinoma of the bladder. J Urol 2003;169:1291-4.

[45] Dyrskjot L, Reinert T, Algaba F, et al. Prognostic impact of a 12-gene progression score in non-muscle-invasive bladder cancer: a prospective multicentre validation study. Eur Urol 2017;72:461-9.

[46] Meeks JJ, Carneiro BA, Pai SG, et al. Genomic characterization of high-risk non-muscle invasive bladder cancer. Oncotarget 2016;7:75176-84.

[47] Pietzak EJ, Bagrodia A, Cha EK, et al. Next-generation Sequencing of nonmuscle invasive bladder cancer reveals potential biomarkers and rational therapeutic targets. Eur Urol 2017;72:952-9.

[48] Sjödahl G, Lovgren K, Lauss M, et al. Toward a molecular pathologic classification of urothelial carcinoma. Am J Pathol 2013;183:681-91.

[49] Choi JW, Kim Y, Lee JH, Kim YS. CD74 expression is increased in high-grade, invasive urothelial carcinoma of the bladder. Int J Urol 2013;20:251-5.

[50] Stern JB. Unusual benign bladder tumor of Brunn nest origin. Urology 1979;14:288-9. [51] Lin O, Cardillo M, Dalbagni G, Linkov I, Hutchinson B, Reuter VE. Nested variant of urothelial carcinoma: a clinicopathologic and immunohistochemical study of 12 cases. Mod Pathol 2003;16:1289-98.

[52] Venyo AK. Microcystic variant of urothelial carcinoma. Adv Urol 2013;2013:654751.

[53] Brimo F, Dauphin-Pierre S, Aprikian A, et al. Inverted urothelial carcinoma: a series of 12 cases with a wide morphologic spectrum overlapping with the large nested variant. Hum Pathol 2015;46:1506-13.

[54] Cox R, Epstein JI. Large nested variant of urothelial carcinoma: 23 cases mimicking von Brunn nests and inverted growth pattern of noninvasive papillary urothelial carcinoma. Am J Surg Pathol 2011;35:1337-42.

[55] Comperat E, McKenney JK, Hartmann A, et al. Large nested variant of urothelial carcinoma: a clinicopathological study of 36 cases. Histopathology 2017;71:703-10. 
Fig. 1 - Comparison of the World Health Organisation (WHO) 1973 and WHO 2004/2016 classification of urothelial carcinoma.

\section{Comparison WHO 1973-2016}

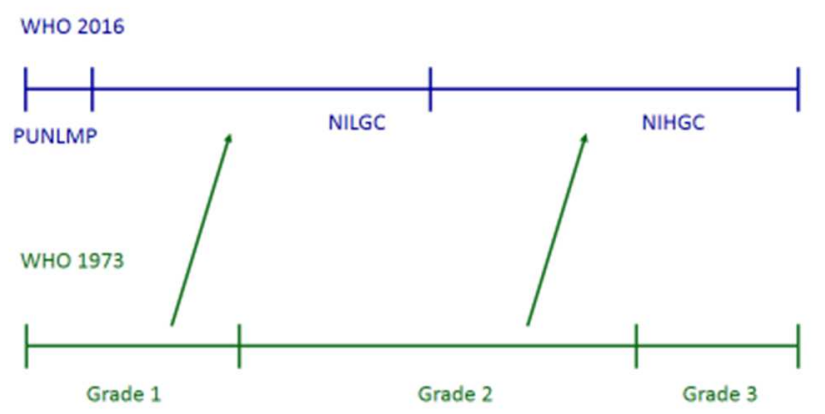

NILGC = noninvasive low-grade papillary urothelial carcinoma; NIHGC $=$ noninvasive high-grade papillary urothelial carcinoma; PUNLMP = papillary urothelial neoplasms of low malignant potential. 
Fig. 2 - No atypia are seen in a large-nest urothelial carcinoma invading the muscle.

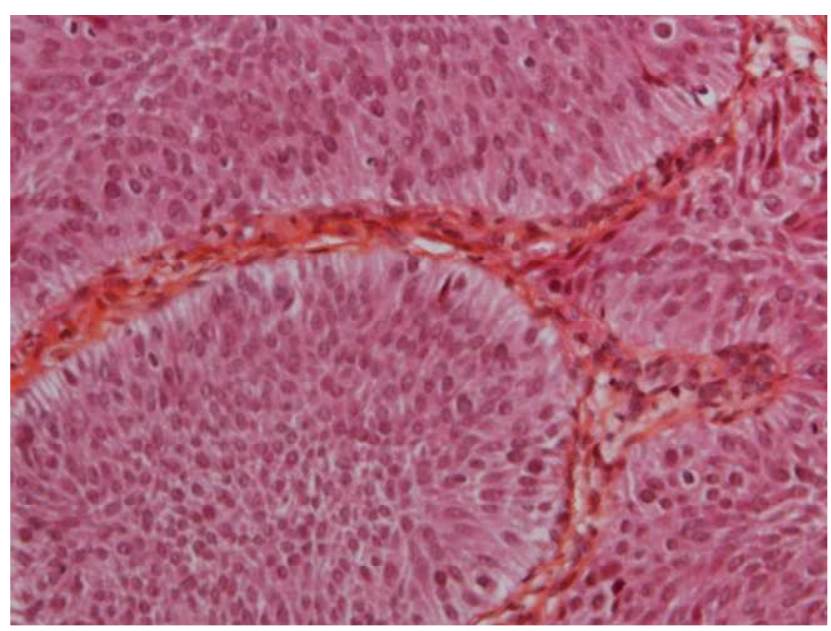


Fig. 3 - Large nests can be seen here dividing and invading the detrusor muscle.

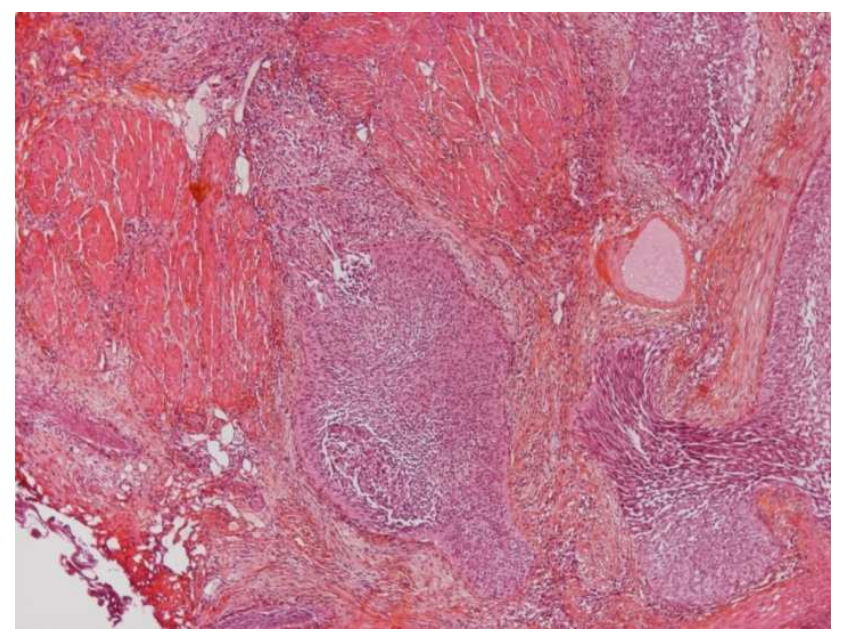


Table 1 - The World Health Organisation 1973 and World Health Organisation 2004/16 classification of urothelial bladder tumours

\begin{tabular}{|c|c|c|c|}
\hline Tumour grade & 1 & 2 & 3 \\
\hline Description 1973 & $\begin{array}{l}\text { Slender papillae in an } \\
\text { orderly arrangement }\end{array}$ & $\begin{array}{l}\text { Atypia can be more or } \\
\text { less marked, increased } \\
\text { urothelial layers }\end{array}$ & $\begin{array}{l}\text { Marked atypia, loss } \\
\text { of polarity, mitosis }\end{array}$ \\
\hline Micrograph & & & 4 \\
\hline $\begin{array}{l}\text { Biological } \\
\text { potential }\end{array}$ & $\begin{array}{l}\text { Papillary neoplasia of } \\
\text { low malignant potential }\end{array}$ & Low & High \\
\hline $\begin{array}{l}\text { Description } \\
2004 / 16\end{array}$ & $\begin{array}{l}\text { Slim papillae without } \\
\text { atypia, no thickening of } \\
\text { the urothelium }\end{array}$ & $\begin{array}{l}\text { Papillae have increased } \\
\text { layers, atypia is rare, } \\
\text { polarity conserved }\end{array}$ & $\begin{array}{l}\text { Major atypia, major } \\
\text { architecture } \\
\text { destroyed }\end{array}$ \\
\hline Micrograph & & & \\
\hline
\end{tabular}

Table 2 - Recurrence and progression rates of papillary neoplasia of low malignant potential

\begin{tabular}{|l|l|l|l|l|l|}
\hline Reference & $\begin{array}{l}\text { Pan et al } \\
2010[11]\end{array}$ & $\begin{array}{l}\text { Compérat et al } \\
2015[12]\end{array}$ & $\begin{array}{l}\text { Pich et al } \\
2001[13]\end{array}$ & $\begin{array}{l}\text { Samaratunga } \\
\text { et al 2002 } \\
{[14]}\end{array}$ & $\begin{array}{l}\text { Holmäng et al } \\
2002[15]\end{array}$ \\
\hline$n$ & 212 & 42 & 19 & 26 & 68 \\
\hline $\begin{array}{l}\text { Recurrence rate } \\
(\%)\end{array}$ & 18 & 17 & 47 & 7 & 0 \\
\hline $\begin{array}{l}\text { Progression } \\
\text { rate }(\%)\end{array}$ & $8 \%$ & $10 \%$ & Not done & $8 \%$ & $14.7 \%$ \\
\hline
\end{tabular}


${ }^{a}$ International Bladder Cancer Group defines nonmuscle invasive bladder cancer progression as an increase in T stage from carcinoma in situ or Ta to T1 (lamina propria invasion), development of $\mathrm{T} 2$ or greater or lymph node metastasis.

Table 3 - Comparison of interobserver variability between the World Health Organisation (WHO) 1973 and WHO 2004/2016 classification systems [2,3,8]

\begin{tabular}{|l|l|l|l|}
\hline WHO 2004/2016 & $\begin{array}{l}\text { Interobserver } \\
\text { variability (\%) }\end{array}$ & WHO 1973 & $\begin{array}{l}\text { Interobserver } \\
\text { variability (\%) }\end{array}$ \\
\hline PUNLMP & $12-39$ & G1 & $48-50$ \\
\hline NILGC & $27-63$ & G2 & $13-69$ \\
\hline NIHGC & $21-67$ & G3 & $18-56$ \\
\hline
\end{tabular}

NIHGC $=$ noninvasive papillary urothelial carcinoma high grade $;$ NILGC $=$ noninvasive papillary urothelial carcinoma low grade; PUNLMP = papillary urothelial neoplasms of low malignant potential. 